\title{
EVALUACIÓN DE LA CARGA POSTURAL PROVOCADA POR LAS CONDICIONES ACTUALES DE LAS ESTACIONES DE TRABAJO DE LOS CAJEROS, EN LAS AGENCIAS DE UNA ENTIDAD FINANCIERA
}

\author{
Esteban Narváez S, ²Víctor Arias B.
}

\author{
'Universidad Tecnológica Equinoccial, Quito, Ecuador, emnarvaes@yahoo.com \\ 2Universdad Tecnológica Equinoccial, Quito, Ecuador, abvh42914@ute.edu.ec
}

Recepción/Received: 22, 09, 2014

Aceptación/Accepted: 22, 10, 2014

Publicado/Published: 12, 12, 2014

\section{RESUMEN}

El objetivo principal de la presente investigación fue evaluar la carga postural provocada por las condiciones físicas de las estaciones de trabajo de los cajeros, en diez agencias de una entidad financiera en la ciudad de Quito. La muestra estuvo compuesta por 43 personas, quienes llenaron un cuestionario general para determinar molestias músculo esqueléticas. Posteriormente se realizó la evaluación de la carga postural a trece usuarios del total de la muestra mencionada, utilizando los métodos OWAS y RULA.

Se encontró que el 77\% de participantes presentaban molestias principalmente en el cuello y zonas altas y bajas de la espalda. El 60\% de las posturas evaluadas con el método OWAS reflejaron niveles donde existe la posibilidad de causar daño al usuario. El método determinó las zonas de riesgo en la estación de trabajo.

El 37\% de posturas evaluadas con el método RULA, exigieron un estudio a profundidad y corrección de posturas, mientras que el $25 \%$ de posturas analizadas, demandaron cambios urgentes en el puesto de trabajo.

Se propone al final, realizar la reingeniería de las estaciones de trabajo enfatizando las mejoras en las zonas críticas para controlar los riesgos, plantear programas de promoción de la actividad física, acompañados de capacitación sobre higiene postural, vigilancia de la salud y ergonomía participativa.

\section{Palabra Clave:}

Cajeros de banco, carga postural, ergonomía, OWAS, RULA.

\section{SUMMARY}

The main objective of the investigation was to evaluate the postural overload caused by the physical infrastructure of the working stations assigned to bank tellers of ten agencies of a financial institution in the city of Quito. A general musculoskeletal discomfort survey was conducted to a sample of workers composed by 43 subjects. A postural overload evaluation, using the methods OWAS and RULA, was applied to thirteen bank teller workstation users.

This study recorded that $77 \%$ of participants show discomfort mainly in the neck and low and upper back. $60 \%$ of the postures evaluated with OWAS method reflected risk levels of physical injures on users. The application of this method helps to determine risk zones on bank teller working stations. The 37\% of postures evaluated with RULA method showed the need of profound research and postural correction, whereas $25 \%$ of analyzed postures demanded urgent changes on the working place. A reengineering of the working stations was suggested, emphasizing the improvements on risk zones to reduce the postural overload. The study also suggests the design of programs, these programs contain: the promotion of the physical activity, training on postural hygiene, vigilance of health and establishing ergonomic participative groups.

\section{Keyword:}

Bank tellers, postural overload, ergonomics, OWAS, RULA. 


\section{INTRODUCCIÓN}

Aparentemente, las contadas y repetidas tareas que desarrolla un cajero podrían parecer, a simple vista, como sencillas de realizar empleando pocos recursos físicos y mentales; no obstante, uno de las principales áreas del Front Office de una institución financiera es la de cajas donde diariamente se pueden dar más de 800 transacciones financieras (Cuanswers, 2014). De ahí que las instituciones financieras dediquen esfuerzos considerables en el diseño e importantes inversiones económicas para poder ofrecer al cliente seguridad, agilidad, buena imagen y versatilidad en las diferentes operaciones financieras que se efectúan en el área de cajas.

Dentro del conjunto de actividades que se efectúan, entre la concepción de un puesto de trabajo y su realización, la ergonomía, como ciencia, aporta una amplia base de información al proceso de diseño. La omisión de dicha información, puede generar en el usuario, alteraciones relacionadas con su salud, además de la reducción de la calidad del producto y disminución del nivel de productividad (Rescalvo, De la Fuente, 2004; Camargo, 2007). Parte de las alteraciones de la salud son aquellas afecciones al sistema musculoesquelético ocasionadas por la demanda física exigida por las tareas que se desarrollan en las estaciones de trabajo diseñadas para los cajeros (Roelof, Straker, 2002).

Sobre el análisis de la demanda física que implica el trabajo en el área de cajas de las instituciones financieras existen pocos estudios internacionales que demuestren el nivel de riesgo al que están expuestos. En el entorno nacional existe una limitada cantidad de estudios de riesgo laborales en instituciones financieras, los cuales determinan como principales riesgos aquellos de origen psicosocial y ergonómicos (Suasnavas, 2007).

En tal sentido, la presente investigación persigue el establecimiento de un precedente en el estudio y análisis de las estaciones de trabajo de una entidad financiera, con el fin de evaluar la carga postural y las molestias ocasionadas por las mismas.

\section{MÉTODOLOGÍA}

La investigación, de tipo descriptivo transversal, utilizó un universo total de personas que estuvo compuesto por 43 colaboradores, los cuales desempeñaban el cargo de Asesor Integral en 10 agencias de una institución financiera de la Ciudad de Quito. Los trabajadores estudiados desempeñaban sus actividades en jornadas laborales de 8 horas en turnos rotativos.

Una vez definidas las variables de la investigación, se diseñó una encuesta compuesta de las siguientes partes: datos generales, esquema de definición de las zonas del cuerpo y cuestionario general sobre molestias en el aparato locomotor. La última parte utilizó como base una versión traducida al español del Cuestionario Nórdico para el Análisis de los Síntomas Musculoesqueléticos desarrollado por Kuorinka, Jonsson, Kilbom, Vinterberg, BieringSørensen, Andersson, Jørgensen (1987) y descrito por INSHT (2002). Previamente se aplicó una encuesta piloto a nueve personas con el fin de comprobar la operatividad, estructura, entendimiento y pertinencia de los elementos del instrumento diseñado. Luego de definir la herramienta de estudio, que incluyó los cambios sugeridos, la encuesta, finalmente, fue aplicada a la totalidad del universo estudiado.

Con el fin de determinar la precisión y consistencia de los puntajes obtenidos en la aplicación de la encuesta, se utilizó el método de consistencia interna o Alfa de Cronbach. Después de haber aplicado la fórmula que recomienda el método, la misma arrojó los siguientes resultados: con el cálculo de 39 ítems, se obtuvo un valor de 0,8. Dentro de los rangos que el método sugiere, el valor obtenido se encuentra en la escala definida como "Alta"; por lo tanto, el instrumento utilizado es fiable, pues permite que las mediciones de las variables sean estables y consistentes.

De forma paralela a la aplicación de la encuesta, se procedió a realizar un levantamiento planimétrico de las medidas de una estación de trabajo tipo, en una de las agencias bancarias. En la Fig.1 se muestra la planta del levantamiento realizado.
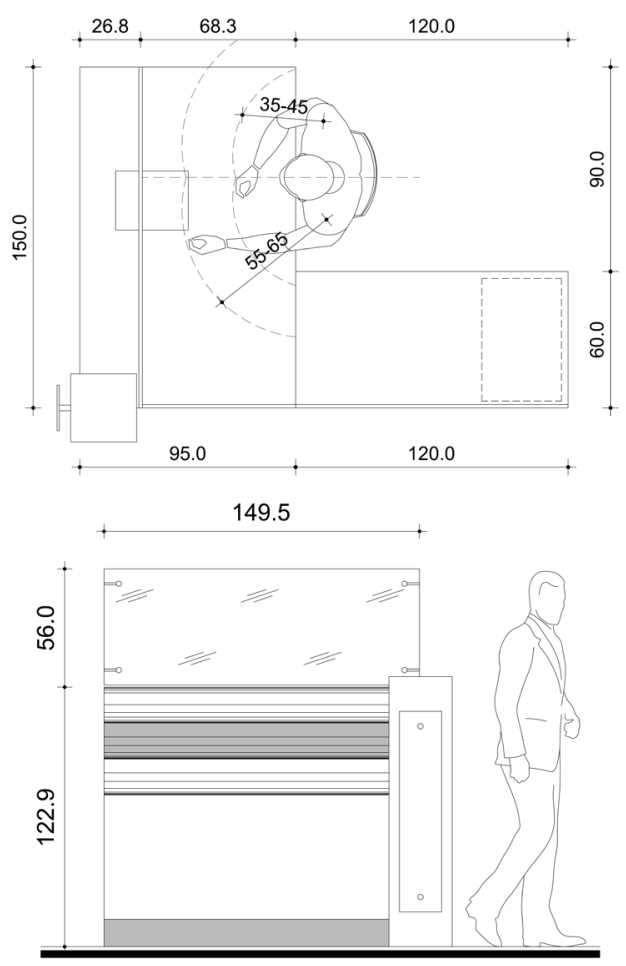

Figura $N^{0}$ 1. Planta y alzado de las estaciones de trabajo con medidas de levantamiento planimétrico y alcances máximos. 
Posteriormente se estableció un cronograma de visita a las 10 agencias bancarias de la institución financiera estudiada, con el fin de entrevistar a los colaboradores y proceder a la elaboración de videos y fotografías sobre el desarrollo de las actividades en cada una de las estaciones de trabajo.

Con la información recolectada se procedió a la edición de los videos y fotografías con el fin de determinar las posturas que implican una mayor carga postural. Luego se procedió a analizar las posturas seleccionadas de acuerdo con los procedimientos de evaluación ergonómicos recomendados en los Métodos OWAS y RULA (descritos por Diego y Asencio, 2012) para determinar el nivel de riesgo al que estaban expuestos los trabajadores.

\section{RESULTADOS Y DISCUSIÓN}

El ejercicio de planimetría para el levantamiento de medidas de la estación asignada para el trabajador permitió la obtención de datos que fueron contrastados con los criterios de los diferentes autores consultados. En la Tabla 1 se puede observar cuál de los rangos reales cumple con los valores teóricos.

Tabla № 1. Comparativo de los parámetros obtenidos en la medición con la normativa técnica.

Fuente: Varios autores

Elaborado por: Esteban Narváez

\begin{tabular}{|c|c|c|c|}
\hline NORMA & RANGO & CUMPLE & $\begin{array}{l}\text { NO } \\
\text { CUMPLE }\end{array}$ \\
\hline \multicolumn{3}{|c|}{ PARÁMETRO: ALTURA DEL PLANO EN TRABAJO LIGERO } & $110 \mathrm{~cm}$. \\
\hline $\begin{array}{l}\text { - Criterio Ayoub para } \\
\text { mujeres (Rescalvo y De la } \\
\text { Fuente, 2004) }\end{array}$ & $87-98$ & & $x$ \\
\hline $\begin{array}{l}\text { - Criterio Gradjean para } \\
\text { mujeres (INSHT, 1997) }\end{array}$ & $85-90$ & - & X \\
\hline - Criterio CCSSO (1998) & $85-110$ & & \\
\hline $\begin{array}{l}\text { - Criterio Maradeí et al. } \\
\text { (2009) }\end{array}$ & $96,2-116,7$ & - & \\
\hline \multicolumn{3}{|c|}{ PARÁMETRO: ANCHO DEL PLANO DE TRABAJO } & $150 \mathrm{~cm}$. \\
\hline $\begin{array}{l}\text { Criterio De Pedro y } \\
\text { Gómez (2001) }\end{array}$ & 150 & - & \\
\hline Criterio INSHT (1990) & 120 & - & \\
\hline $\begin{array}{l}\text { Criterio Barnes y Squires } \\
\text { (Rescalvo y De la Fuente, } \\
\text { 2004) }\end{array}$ & 150 & $\bullet$ & \\
\hline \multicolumn{3}{|c|}{ PARÁMETRO: PROFUNDIDAD DEL PLANO DE TRABAJO } & $95 \mathrm{~cm}$. \\
\hline $\begin{array}{l}\text { Criterio De Pedro y } \\
\text { Gómez (2001) }\end{array}$ & 90 & & $x$ \\
\hline Criterio INSHT (1990) & 80 & & $x$ \\
\hline $\begin{array}{l}\text { Criterio Farley (Farrer et } \\
\text { al., 1994) }\end{array}$ & $59,7-67,3$ & & $x$ \\
\hline $\begin{array}{l}\text { Criterio Barnes y Squires } \\
\text { (Rescalvo y De la Fuente, } \\
\text { 2004) }\end{array}$ & 50.8 & & $x$ \\
\hline $\begin{array}{l}\text { Criterio Maradeí et al. } \\
(2009)\end{array}$ & 52,1 & & $\mathrm{x}$ \\
\hline \multicolumn{3}{|c|}{ PARÁMETRO: ESPACIO DESTINADO PARA LAS PIERNAS (ANCHO) } & $90 \mathrm{~cm}$. \\
\hline $\begin{array}{l}\text { Criterio Rescalvo y De la } \\
\text { Fuente (2004) }\end{array}$ & 60 & • & \\
\hline $\begin{array}{l}\text { Criterio De Pedro y } \\
\text { Gómez (2001) }\end{array}$ & 60 & - & \\
\hline Criterio INSHT (1990) & 70 & $\bullet$ & \\
\hline \multicolumn{3}{|c|}{ PARÁMETRO: ESPACIO DESTINADO PARA LAS PIERNAS (PROFUNDIDAD) } & $68,3 \mathrm{~cm}$. \\
\hline $\begin{array}{l}\text { Criterio Rescalvo y De la } \\
\text { Fuente (2004) }\end{array}$ & 60 & - & \\
\hline $\begin{array}{l}\text { Criterio De Pedro y } \\
\text { Gómez (2001) }\end{array}$ & $80-90$ & & X \\
\hline Criterio INSHT (1990) & 70 & & $x$ \\
\hline
\end{tabular}

La encuesta aplicada a las 43 personas permitió caracterizar a la población de estudio de la siguiente manera: la edad promedio de la población estudiada fue de 28 años y en ella predomina el género femenino, con un 84\%, mientras que el género masculino estuvo representado por un 16\%. A los encuestados se les consultó sobre su peso y altura. Relacionando estas dos variables se obtuvo el índice de masa corporal y se encontró que más de la mitad de los colaboradores se encuentran en un rango de normalidad, mientras que un $23,26 \%$ se encuentran en la zona de preobesidad. Cuando se consultó sobre la lateralidad que manejan, 39 de los 43 encuestados respondieron que manejaban preferentemente su lado derecho.

Dentro del cuestionario se incluyeron consultas sobre la práctica de pausas activas, sobre lo cual el 60\% respondió que no realizaba esta práctica y tan solo el $40 \%$ efectuaba interrupciones en sus actividades para realizar ejercicios de relajación. En la Fig. 2 se detalla el tipo de actividades que se realizan durante las pausas activas, siendo la principal el cambio de posición o postura:

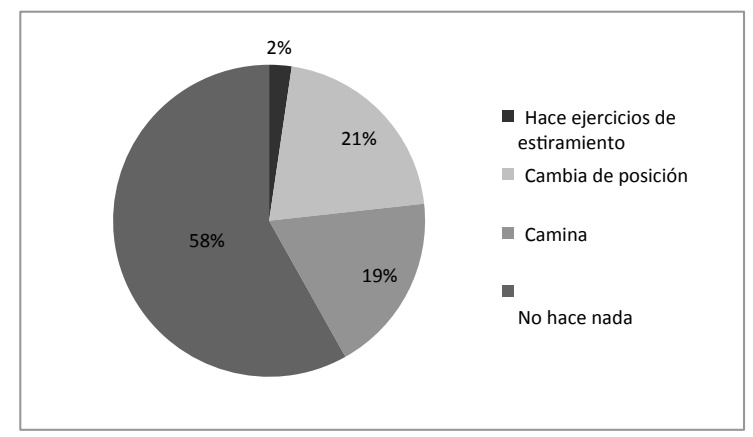

Figura $N^{\circ} 2$. Tipos de pausas activas que se practican.

Al ser consultados sobre el tiempo que llevaban desempeñando las actividades entre el área de cajas y el servicio al cliente, se encontró que más del 60\% llevaba trabajando más de un año sin llegar a cumplir los cinco años como empleados en la empresa. La mayoría de trabajadores cumple con el horario exigido por la ley, esto es, el de ocho horas.

Para finalizar la primera parte del cuestionario se pidió la opinión sobre la comodidad de la estación de trabajo y la mayoría afirmó que el área de trabajo resultaba incómoda. En la Fig. 3 se ilustra la opinión consultada.

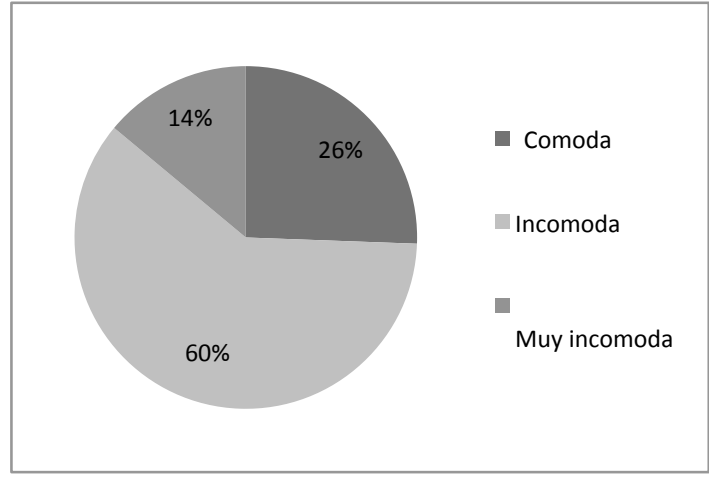

Figura № 3. Distribución porcentual sobre opinión de la estación de trabajo actual. 
En la segunda parte del instrumento utilizado se estudiaron las molestias músculo-esqueléticas de los encuestados en el último año; el 58,14\% aseguró tener molestias en la zona del cuello y en la zona superior de la espalda. En un menor porcentaje, las molestias se mostraron en un 41,86\% en la zona inferior de la espalda. En la Fig.4 se detallan los resultados obtenidos:

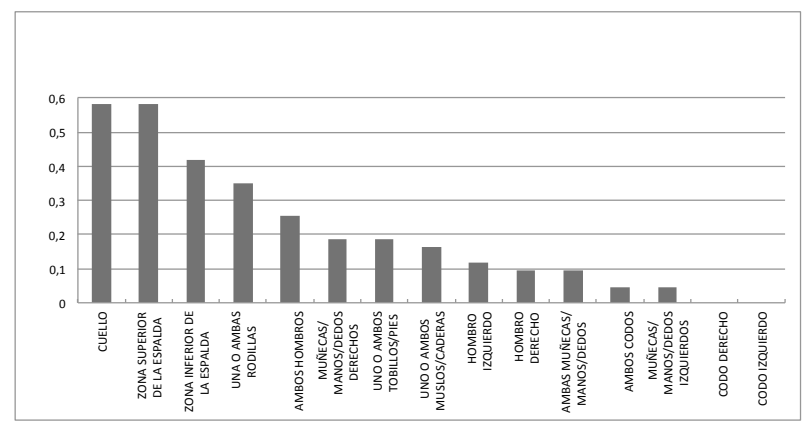

Figura $N^{\circ} 4$. Distribución porcentual de molestias por ubicación anatómica en los últimos 12 meses.

Cuando se consultó si se había tenido molestias recientes, como se muestra en la Fig. 5, estas se presentaron en un $44,19 \%$ en la zona superior de la espalda; $41,86 \%$ en el cuello; $27,91 \%$ en la zona inferior de la espalda, y $25,58 \%$ en una o ambas rodillas.

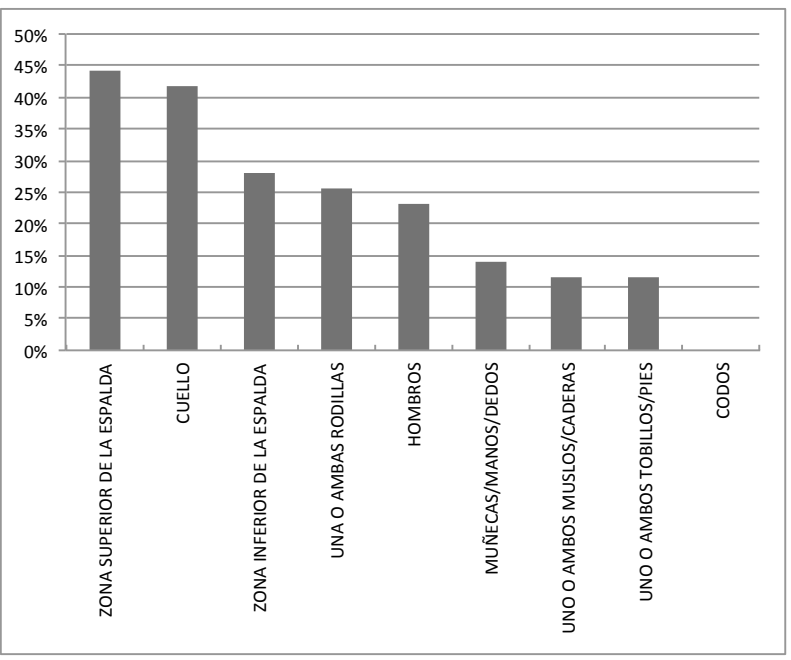

Figura $N^{\circ} 5$. Distribución porcentual de molestias por ubicación anatómica en los últimos 7 días.

El primer método de evaluación ergonómica que se aplicó para valorar el riesgo de las posturas generadas por el uso de las estaciones de cajeros fue el método OWAS, con el que se analizaron 100 posturas. Dentro de las cuatro categorías de riesgo que propone el método, se encontró que el $60 \%$ de posturas analizadas se situaban dentro de la Categoría 2, que determina posturas con posibilidad de causar daño al sistema músculo-esquelético, por lo que se requieren acciones correctivas en un futuro cercano. El $27 \%$ de posturas analizadas se enmarcaron dentro de la Categoría 3, que determina posturas con efectos dañinos sobre el sistema músculo-esquelético, por lo que se requiere acciones correctivas lo antes posible. En la Fig. 6 se ilustra los resultados obtenidos.

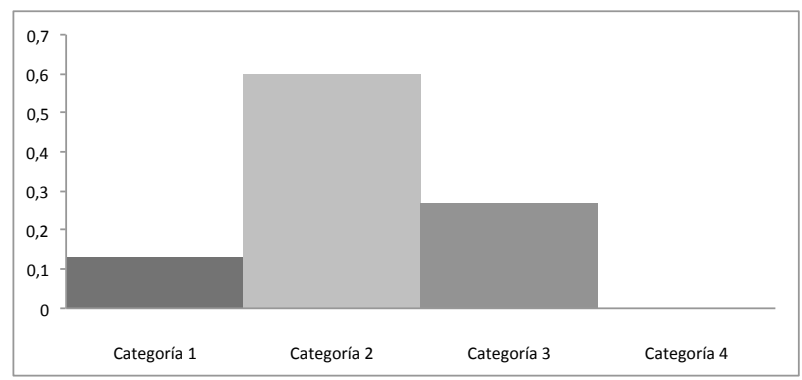

Figura № 6. Categorización de riesgos de posturas según método de evaluación OWAS.

Para el análisis de las 100 posturas, se las agrupó de acuerdo con las diferentes fases, según las tareas desempeñadas por los usuarios. De este procedimiento se obtuvieron los siguientes resultados.

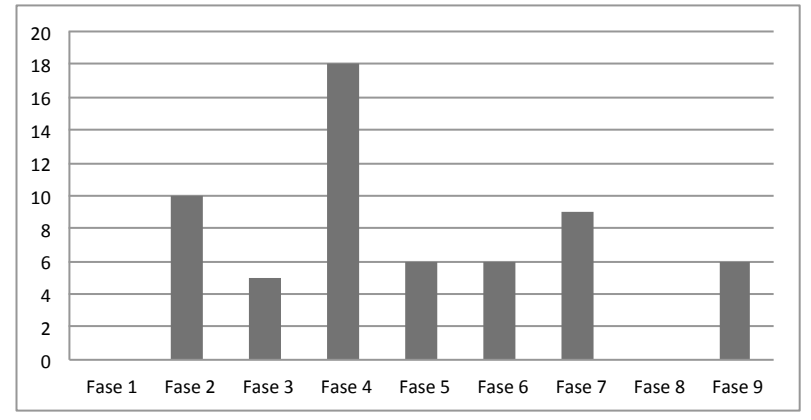

Figura $N^{\circ} 7$. Frecuencia de posturas en fases de más riesgo, Cat. 2.

En la Fig. 7 se muestran las posturas agrupadas dentro de la Categoría 2, que determina posturas con posibilidad de causar daño al sistema músculo-esquelético, por lo que se requieren acciones correctivas en un futuro cercano; 18 posturas estuvieron en fase 4, correspondiente al conteo del dinero. También existen 10 posturas que se agruparon en fase 2, correspondiente a la recepción de la documentación del cliente, y 9 posturas se agruparon en fase 7 , correspondiente al reconteo del dinero.

La Fig.8 muestra las posturas agrupadas dentro de la Categoría 3, que determinó efectos dañinos sobre el sistema músculo-esquelético, por lo que se requieren acciones correctivas lo antes posible. 10 posturas se agruparon en la fase 4, correspondiente al conteo del dinero. Tan solo 5 posturas se agruparon en fase 5, correspondiente a la actualización de libreta de ahorros.

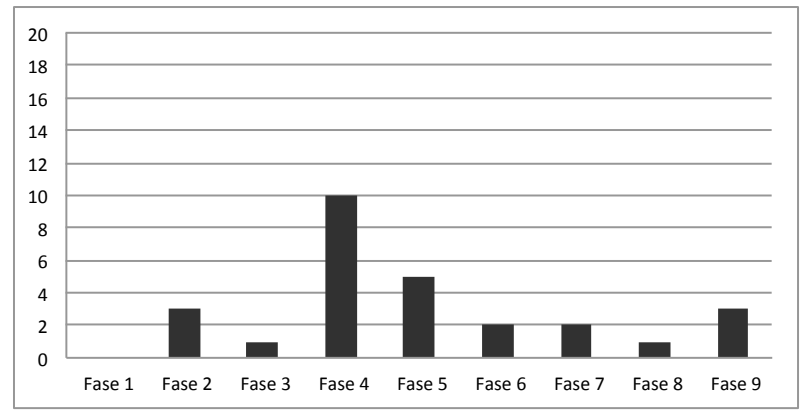

Figura $N^{\circ} 8$. Frecuencia de posturas en fases de más riesgo, Cat. 3. 
El segundo método aplicado para evaluar las posturas forzadas en las estaciones de cajeros, fue el método RULA, con el que se evaluaron 49 posturas consideradas como aquellas de mayor carga postural para los trabajadores investigados. Dentro de los cuatro niveles de riesgo que propone el método, se encontró que el 37\% de posturas analizadas obtuvo una puntuación final entre 3 y 4 puntos o Nivel 2, con lo cual se establece que hay la posibilidad de requerirse cambios en la tarea y es conveniente profundizar en el estudio. En igual porcentaje se ubicaron aquellas posturas que obtuvieron una puntuación entre 5 y 6 puntos, calificación que determina el Nivel 3 del método, y establece efectuar un estudio en profundidad, corrigiendo la postura lo antes posible. Finalmente se registró que el 25\% de las posturas evaluadas se ubicó en el Nivel 4, con una puntuación final de 7 puntos. En este nivel, el método establece cambios urgentes en el puesto o tarea. En la Fig. 9 se ilustran, de igual manera, los resultados obtenidos.

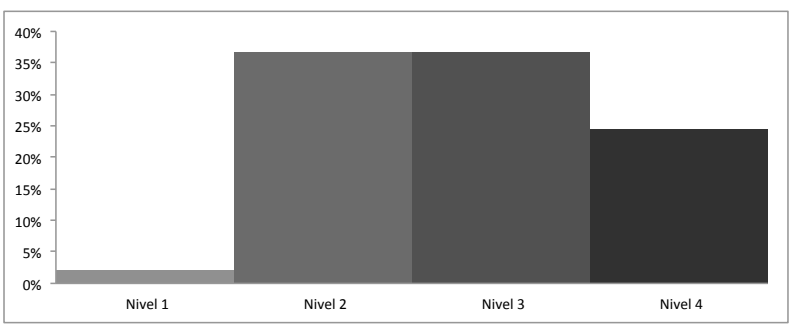

Figura $N^{\circ}$ 9. Niveles de riesgos de las posturas evaluadas, según método de evaluación RULA.

En lo referente a la actividad física laboral, se encontró que el $60 \%$ de encuestados no realiza pausa activas, lo que es corroborado con el 58\% de personas que no realizan ninguna actividad física durante sus pausas. La exposición al riesgo causado por cargas posturales se puede ver reducida si se incorpora a las rutinas laborales un programa periódico y sistemático de pausas activas que incluya la capacitación del personal y la asignación de responsables según lo recomendado por León (2011) dentro de las intervenciones ergonómicas.

Al igual que el estudio planteado por Roelof, Straker (2002), los resultados de la presente investigación (77\%) coinciden en que la postura más cómoda para el desarrollo de las actividades en cajas es aquella que alterna la posición de sentado y parado, lo que se debería tomar en cuenta en cualquier recomendación sobre reingeniería de las estaciones.

En la segunda parte del cuestionario, donde la atención de estudio se centra en las molestias corporales específicas, los resultados arrojaron como las zonas corporales más afectadas las siguientes: zona superior de la espalda, zona del cuello y zona inferior de la espalda. Esto coincide con el estudio realizado por López, Aires y Bolta (2000), donde se establecen como razones principales de ausentismo, la aparición de dolencias físicas tales como dolores de espalda, dolores cervicales y hernias.

Con lo que respecta a la evaluación ergonómica de las cargas posturales, mediante el uso de los métodos OWAS y RULA respectivamente, los resultados sobre la valoración de las posturas que implican riesgo, indican, además de la gravedad, las zonas de la estación de trabajo donde se producen dichas posturas: zona de cajonera metálica, zona de bandeja pasa documentos y zona de impresora. Este aporte que realiza la presente investigación, puede ayudar a definir las zonas, el diseño y la reingeniería de las estaciones, sobre las cuales se debería tomar mayor énfasis.

\section{CONCLUSIONES Y RECOMENDACIONES}

Por medio de la utilización de métodos de evaluación ergonómica probos, se pudo establecer que los usuarios de las estaciones de cajas estuvieron expuestos a una carga postural considerable, ocasionada por la infraestructura física de las estaciones de trabajo.

El análisis de los procedimientos y tareas que realizan los cajeros de la entidad financiera estudiada, dio paso a la definición de actividades o fases, que al ser evaluadas, permitieron a su vez, definir las zonas críticas de las estaciones de trabajo que provocan la carga postural de sus usuarios: zona de cajonera metálica, zona de bandeja pasa documentos y zona de impresora. Con la identificación de las zonas críticas donde se producen cargas posturales, se podría plantear reingenierías específicas, tomando en cuenta datos antropométricos provenientes de estudios realizados en la población latinoamericana (Maradeí, Espinel, Peña, 2009) y las medidas sugeridas por los autores citados en la presente investigación.

La valoración obtenida en la aplicación de los métodos de evaluación de las posturas forzadas indicó que se tendrán que tomar acciones correctivas a corto plazo, con el fin de modificar la postura de los usuarios, y también realizar cambios en la reingeniería de la estación de trabajo, en el mismo lapso. Parte de los cambios podrían ser la dotación de sillas más resistentes al uso cotidiano, que permitan la alternancia de posturas entre sentado y parado. También se podría incorporar elementos que ayuden al cambio de las condiciones físicas laborales, como la implementación de una tarima que permita la compensación de la diferencia de altura en el interior del área de cajas.

Los resultados obtenidos en la encuesta indicaron una importante presencia de molestias en zonas específicas del cuerpo de los trabajadores (zona superior de la espalda, zona del cuello y zona inferior de la espalda), algunas de las cuales persisten en horarios extra laborales, y las mismas 
están relacionadas con la carga postural producida por la estación de trabajo del área de cajas. Factores externos como el sedentarismo, las pausas pasivas, el sobrepeso y el género podrían incidir en la aparición de las mencionadas molestias. Con el fin de poder reducir el riesgo existente, se podrían implementar Programas de Vigilancia de la Salud que promuevan la actividad física para la prevención de lesiones osteomusculares. Dichos programas podrían incluir capacitación sobre pausas activas, higiene postura y bailo-terapia.

El levantamiento planimétrico de la estación - tipo de cajas permitió determinar medidas que, al ser comparadas con la información obtenida de la revisión bibliográfica, determinó que algunas de ellas se encuentran fuera de los valores recomendados. En futuras reingenierías de las estaciones se podrían tomar como referencia los datos obtenidos en la investigación realizada.

La aplicación del cuestionario sirvió para confirmar el malestar que existió dentro del personal de cajas que consideró como incómodas a las estaciones de las que actualmente disponen. Dicho hallazgo podría afectar el clima laboral de todas las agencias de la entidad financiera motivo del presente estudio. Con el fin de fomentar la participación e inclusión del personal en el proceso de mejora continua, se podría conformar grupos DISERGON para la propuesta de reformas y cambios en la infraestructura y los procedimientos.

La realización de la presente investigación permitió establecer un precedente en la evaluación de riesgos de tipos dis-ergonómicos en las empresas de servicios y más específicamente, en las empresas del sector financiero.

\section{REFERENCIAS BIBLIOGRÁFICAS}

Asensio, S. Basante, M. Diego, J. (2012). Evaluación Ergonómica de Puestos de Trabajo. (Primera Edición).Madrid: Ediciones Paraninfo

Camargo, N. (2007). Diseño industrial y ergonomía. Sociedad de Ergonomistas de México: IX Congreso Internacional de Ergonomía Semac y XIII Reunión Binacional De Ergonomía México-E.U.A.

Centro Canadiense de Seguridad y Salud Ocupacional (CCSSO). 1998. Trabajo de Pie - Información Básica. Recuperado en agosto 2013 de http:// www.ccsso.ca/oshanswers/ergonomics/standing/ standing_basic.html

Cuanswers. (2014). Understanding CU*BASE Teller: Analysis Reports. Teller Transaction Monitoring. Recuperado en febrero 2014 de http://cuanswers. com/pdf/cb_ref/T-TellerTransactionMonitoring.pdf

De Pedro, O. Gómez, M. (2001). Temas de Ergonomía y prevención: Ergonomía 4, El Trabajo en Oficinas.
(Primera Edición). Barcelona: Universidad Politécnica de Cataluña.

Farrer, F. Minaya, G. Escalante, J. Ruiz, M. (1994). Manual de Ergonomía. Madrid: Editorial MAPFRE S.A. Fundación MAPFRE.

Instituto Nacional de Seguridad e Higiene en el Trabajo (INSHT). (1990). Nota Técnica de Prevención No. 242: Ergonomía: análisis ergonómico de los espacios de trabajo en oficinas. España: Ministerio de Trabajo e Inmigración, Instituto Nacional de Seguridad e Higiene en el Trabajo.

Instituto Nacional de Seguridad e Higiene en el Trabajo (INSHT). (1997). Nota Técnica de Prevención No. 452: Evaluación de las condiciones de trabajo, carga postural. España: Ministerio de Trabajo e Inmigración, Instituto Nacional de Seguridad e Higiene en el Trabajo.

Instituto Nacional de Seguridad e Higiene en el Trabajo (INSHT). (2002). Ergonomía y Psicosociología Aplicada: Evaluación del riesgo derivado de la carga física mediante el registro de las molestias musculoesqueléticas. Curso de técnico superior en prevención de riesgos laborales. España: Ministerio de Trabajo e Inmigración, Instituto Nacional de Seguridad e Higiene en el Trabajo.

Kuorinka, I. Jonsson, B. Kilbom, A. Vinterberg, H. BieringSørensen, F. Andersson, G. Jørgensen, K. (1987). Standardised Nordic questionnaires for the analysis of musculoskeletal symptoms. Applied Ergonomics. Butterworth \& Co.

León, M. (2011). Ergonomía participativa en cajeras de una gran tienda. Ciencia \& Trabajo, No. 39, 57-63.

López, J. Aires, R. Bolta, A. (2000). Estudio ergonómico para una cajera de hipermercado. Recuperado en abril 2012 de http://www.ergonautas.upv.es/arttech/ponencias/EA05.pdf

Maradeí, M. Espinel, F. Peña, A. (2009). Datos Antropométricos para el Diseño. (Primera Edición). Bucaramanga: Universidad Industrial de Santander.

Rescalvo, F. De la Fuente, J. (2004). Ergonomía y Salud, Parte I y II. Junta de Castilla y León, Consejería de Economía y Empleo, Dirección General de Trabajo y Prevención de Riesgos Laborales.

Roelof, A. Straker, L .(2002). The experience of musculoskeletal discomfort amongst bank tellers who just sit, just stand or sit and stand at work. Perth: Curtin University of Technology.

Suasnavas, P. (2007). Diseño e implantación del sistema de gestión de seguridad y salud laboral -modelo Ecuador- para una entidad financiera. Tesis de grado presentada como requisito para la obtención del título de Magíster en Seguridad Salud y Ambiente, Universidad San Francisco de Quito, Quito, Ecuador.

| 36 | REVISTA EÍDOS, diciembre 2014 